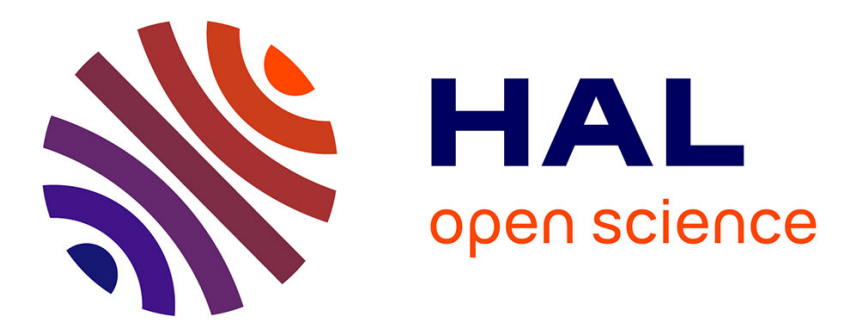

\title{
Using ICT Energy consumption for monitoring ICT usage in an enterprise
}

\author{
Dimitar Minovski, Eric Rondeau, Jean-Philippe Georges
}

\section{To cite this version:}

Dimitar Minovski, Eric Rondeau, Jean-Philippe Georges. Using ICT Energy consumption for monitoring ICT usage in an enterprise. 4th International Conference on Future Internet of Things and Cloud, FiCloud 2016, Aug 2016, Vienne, Austria. hal-01356594

\section{HAL Id: hal-01356594 https://hal.science/hal-01356594}

Submitted on 25 Aug 2016

HAL is a multi-disciplinary open access archive for the deposit and dissemination of scientific research documents, whether they are published or not. The documents may come from teaching and research institutions in France or abroad, or from public or private research centers.
L'archive ouverte pluridisciplinaire HAL, est destinée au dépôt et à la diffusion de documents scientifiques de niveau recherche, publiés ou non, émanant des établissements d'enseignement et de recherche français ou étrangers, des laboratoires publics ou privés. 


\section{Using ICT Energy consumption for monitoring ICT usage in an enterprise}

\author{
Dimitar Minovski \\ University of Lorraine, \\ Vandoeuvre-lès-Nancy, France \\ dimitar.minovski1@etu.univ-lorraine.fr
}

\author{
Eric Rondeau, Jean-Philippe Georges \\ Université de Lorraine, CRAN, UMR 7039, Campus \\ Sciences, BP 70239, Vandoeuvre-lès-Nancy Cedex, 54506, \\ France \\ CNRS, CRAN, UMR 7039, France \\ \{eric.rondeau, jean-philippe.georges\}@univ-lorraine.fr
}

\begin{abstract}
Energy efficient policies are being applied to network protocols, devices and classical network management systems (NMS). Researchers have already studied in depth each of those fields, including for instance a long monitoring processes of various number of individual ICT equipment from where power models are constructed. With the development of smart intelligent meters and emerging protocols such as SNMP and NETCONF, currently there is an open field to couple the power models, translated to the expected behavior of the devices, with the realtime energy measurements. The goal is to derive a comparison on the power data between those parameters in the direction of detection for possible deviations on the expected results. The logical assumption is that a detected fault in the usage of a particular device will not only increase its own energy usage, but also may cause additional consumption on the other devices part of the network. This paper shows the opportunities to use power consumption monitoring of an ICT infrastructure as a substitute for the classical NMS. A platform is developed to monitor and analyze the retrieved power data of a simulated enterprise ICT infrastructure. Moreover, smart algorithms are developed which are aware of the different states that are occurring on each device during their typical use phase. Hence, the platform is able to detect and isolate possible anomalies, for instance improper use of the networking devices. The produced results are obtained and validated with the use of Cisco switches and routers, Dell Precision stations and Raritan PDU as part of the monitored infrastructure.
\end{abstract}

Keywords-Energy efficiency; Network management system; Energy metrics and benchmarks; Performance evaluation and modelling; Performance monitoring; Fault detection and isolation;

\section{INTRODUCTION}

The energy-efficient network infrastructures have recently become a hot topic in the business world, as the concept of Green IT strives to reduce the overall operational costs, but in the same time also to eliminate the inefficiencies from the enterprises' IT systems. The network infrastructures are already massively deployed and even projected to have exponential growth [1] due to the evolution of the Internet, user demands and trending topics such as Internet of Things [3]. Thus, as a shared resource they have to be constantly available, which exacerbates the sustainability issues. Researchers have already proposed different network-wide energy management schemes targeting various areas such as datacenters [4], mobile networks [5] and WANs [6]. However, it is quite challenging to tackle the energy efficiency issues within the households and from small to large enterprises. One obstacle in making enterprise networks more energy efficient is the range of devices from multiple vendors deployed on the network. Also, it is difficult to operate with enterprise networks because of their unpredictable growth, frequently changed topology and architecture, regarding the energy consumption.

Current research and developed Network Management Systems (NMS) are not fully automated when executing energyefficiency policies. Typically they are focused on manual configuration per device, which may be error prone and unlikely to make informed decision for network-wide energy efficiency. Moreover, the classical monitoring part of NMS is based on point-to-point communication with every device on the network, which creates a great deal of traffic and additional burden. Due to improvements in the field of Green IT and smart meters, it is feasible to build a NMS that recons only on power data fetched from the power distribution unit. A pattern for augmenting the values extracted from the power consumption of the network could provide useful information about different network states, for instance detecting changes in the topology. The idea is to use Fault Detection and Isolation (FDI) approach to monitor the network state based on the energy usage. Figure 1 shows that we need two information, a model representing the expected behavior of the devices and the real-time measurements to analyze deviation between the two processes. A deviation corresponds to fault detection in the network, which is in a form of misconfiguration or improper use of the equipment. This means that monitoring the energy consumption could be used not only for Green IT purposes, for raising awareness and reducing the electricity costs, but as well for a classical ICT monitoring system. Also, as the concept of Smart Grid is making use of digital networks to improve the transportation of energy, the work presented in this paper could be explained as the reverse process - how the use of energy could improve the data transport.

The remaining part of this paper is organized as follows: Section II presents the related work, while the Section III presents the objective of the paper. The system is described in Section IV, which includes the architecture for network management, the design of the experiments and the implementation of the developed platform. In Section $\mathrm{V}$ the obtained results are presented and discussed, while the Section VI concludes the paper. 


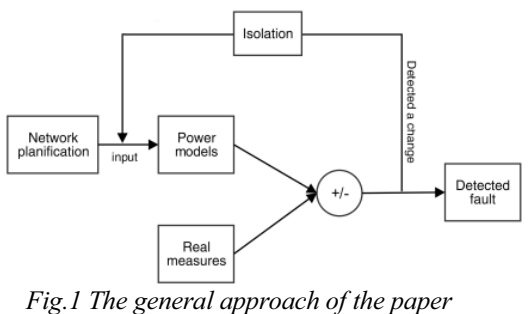

\section{RELATED WORK}

A centralized traffic engineering technique is suggested in [7] for route calculation using a network topology and traffic matrix. The aim is to maximize the energy savings in the network while keeping the performance at a desired level. However, it uses manual configuration through scripts to retrieve the current state of the network, which in a heterogeneous environment with devices from multiple vendors is difficult to manage. Another type of NMS [15] uses standardized network protocols such as Simple Network Management Protocol (SNMP) to manage the power distribution and achieve energy proportionality in a network. The real-time measurements are pulled from each device with point-to-point communication on the network. The introduction of NETCONF/YANG [17] opened new richer ways to perform monitoring and configuration of a network. The advantage of using NETCONF over SNMP is having security and additional capability exchange features, which are necessary for managing complex networks, as well as being more efficient in terms of number of transactions. The experiment in [18] demonstrated that NETCONF is able to configure 100,000 managed objects in a single transaction, while SNMP's best case scenario is 2779 transactions for the same number of managed objects.

The strategy presented in [19] for studying the effects of unknown induced delays in network architecture suggests the use of concepts such as Fault Detection and Isolation (FDI) and Fault Tolerance Control (FTC). The study defines a threshold for the expected delay on the basis of the network characteristic and network calculus theory. A faulty situation is then generated and compared to the defined threshold in order to successfully detect which elements are causing the delays and deals with them in a controlled manner. Similarly, [20] proposes the use of power profiles for each device on the network to determine their expected energy usage under different circumstances. However, the power profiles are considered in special case when the realtime energy measurements are not accessible. They suit as a backup figure to proceed with the FDI's calculations to determine a faulty situation and produce energy efficient policies for the network.

The study by Drouant et al. [8] proposes models that gives a global overview of the impact that the network and the ICT equipment is having on the environment, developing the following equation:

$$
\begin{aligned}
& \mathrm{E}=\mathrm{E}_{\mathrm{m}}+\mathrm{E}_{\mathrm{u}}+\mathrm{E}_{\mathrm{d}} \\
& =\mathrm{E}_{\mathrm{m}}+\int_{t=0}^{\text {end of lifecycle }} P u(t) d t+\mathrm{E}_{\mathrm{d}}
\end{aligned}
$$

Where $E_{m}$ is the energy required for manufacturing and transportation of the equipment, $E_{u}$ is a factor related to the energy consumed during the usage of the equipment, and the energy required to dismantle the equipment is $E_{d}$. For the second part of (1), $P u$ is related to the power consumption by the network architecture during its use phase.

Two strategic approaches exist for modeling the energy consumed during the usage phase of the devices. The first involves a high-level modelling [9] of the whole architecture and the second approach is more precise, meaning that it provides power models for each of the devices part of the network. A high-level model has less interactions with the devices and therefore fails to give accurate estimations how much energy a network architecture consumes at a particular point of time. On the other hand, the use of energy consumption models developed for a particular device would require constant point-to-point interactions and cause certain amount of additional traffic in the network. However, by giving a more precise figure on the expected behavior of the devices the system would be more responsive to minor changes, faults and anomalies, which is the purpose of the experiments part of this paper. Therefore, the power models for Switch [10, 11], Router [12], PC [13] and Access Point [14] are added to the Power Model Registry, as shown on the Figure 3, explained in the next sections.

\section{OBJECTIVE}

The goal is to develop autonomous network management system (NMS) which operates only with the retrieved power consumption data from the devices part of the network infrastructure. The system follows the FDI approach illustrated on the Figure 1 to detect and report for possible faulty situations and anomalies in the usage phase of the ICT equipment. To achieve this, two information as an input parameters are required: (i) power data from real-time measurement procedure and (ii) values for the expected power consumption of each device extracted from the power models.

(i) The process of real-time measurement requires probes and standardized protocols to access the needed metrics which are altogether coupled in a monitoring system. In practice, Raritan Intelligent Power Distribution Unit (PDU) is the monitored equipment which has a predefined energy Management Information Base (MIB) as a hierarchical structure with properties that is pulled out periodically. The monitoring system itself is then able to collect, analyze and modify the information stored in the PDU's MIB through SNMP.

(ii) From the other side, as discussed in the section II, the selected power models represent the expected behavior the equipment. Therefore, currently there is an open field to combine the real-time measures, which are translated to the real behavior of a device, with the developed models. A deviation between the models and the monitored power data is used to detect anomalies and to anticipate fault according to a trend analysis.

\section{A. Building a knowledge base}

The developed platform that incorporates the monitoring system and the power models has to form thorough foreknowledge in order to successfully anticipate and interpret the readings for the energy consumption. Mostly because the power data is just a raw value and does not contain rich information. As suggested by [2], some types of computing 
systems within the enterprise can exhibit large variations on the power data even when comparing two instances of the same device model. More precisely, two Dell Optiplex 760 PCs were observed to have over $40 \%$ discrepancy in their average power draw. To remedy this, the platform has to augment new ways to isolate the problem with the inconsistent energy consumption of the devices, to locate the fault or the anomaly and to classify the problem. One way to deal with this is to predict the fault that may occur during the usage of the equipment. It is important to test the responsiveness of the platform by generating different faults and misconfigurations that may occur during a typical workday in an enterprise network. Thus, closely observe the figures for energy consumption and build a knowledge base. A system with such knowledge comprised with smart algorithms is capable of analyzing the deviations and benchmark the faulty situations. From there a pattern could emerge to later detect the problem of aberrant values regarding the energy consumption of the devices during the real-life usage phase of the equipment.

\section{B. The logic behind the NMS}

The states illustrated on the Figure 2 represent the logic throughout the execution process. Having developed a standalone platform able to monitor the energy consumption as a first state allows further analysis process on the retrieved and stored data. The analysis engine as a second state is tightly coupled with the learning model of the system by developed smart algorithms which are able to detect and identify the changes in the power data. Big part of the analysis process plays the inclusion of the power models [10-14] which are defining the power profiles of the devices and are calculating their expected behavior. The output of the models is then validated with the retrieved real-time measurements and the results are logically passed to the third state, the learning process, where they are further dismantled towards understanding and interpreting the power data values. The emphasis of the third state, the learning process, is given to the simulation of the faults that are most frequent during the regular usage phase of the ICT equipment. The established knowledge base is used as a baseline on which the learning model is constructed. This means that series of experiments have to be conducted for a range of different faults in the interest of acquiring the desired foreknowledge, which is also the key for the isolation part as a last state of the logic depicted on the Figure 2.

Ever since the commencement period of the learning process, the system is constantly updating its knowledge base depending on the behavior of the devices, and by following the figures for the energy consumption it is able to discern the aberrant states of the devices. For instance, a possible overloading on the devices, detecting sleep/hibernate states, physical or logical changes in the configuration of the devices, etc. To have seamless operations and accurate output, the learning process also needs to update the static values in the power models based on the detected new state of a particular device. Namely, as seen on the Figure 1, the formulas in the power models depend on static parameters from the network planification, which are gathered during one-time measurement. This means that the learning model has to re-compute the input parameters in the power models after a detection of a new network state, for instance transiting a switch port from offline

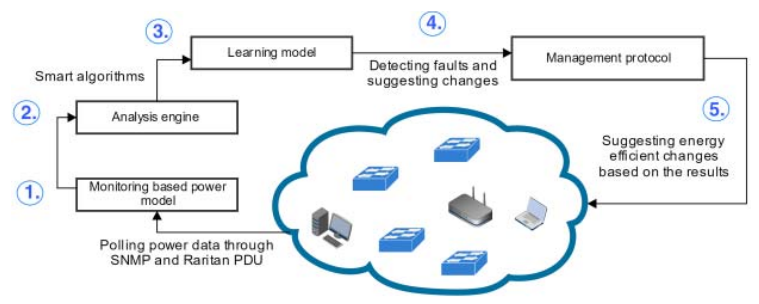

Fig.2 The experiment's logic

to online state. This concludes the final fourth state of the logic behind the experiment, the engine for power management, because the outcome from the previous states results with identifying the changes that happened in the network infrastructure and suggests a recuperation, or a reconfiguration of the network.

\section{SYSTEM DESCRIPTION}

\section{A. System Architecture}

The architecture of the system shown on the Figure 3 consists of subcomponents for each of the main components, namely: (i) Power Model Registry; (ii) Network Management System; and (iii) Equipment. The integrated view of the network infrastructure is the foremost advantage of the architecture, enabling the enforcement of energy-efficient policies while having a minor impact on the overall consumption and the traffic flowing through the network.

(i) The Power Model Registry includes a set of static parameters that define a power profile extracted from the hardware pieces such as the chassis, the forwarding engine/cpu or the cards/ports of the devices. Among those parameters there is a derived scaling factor for energy consumption dependent on the load. For instance, a power model would be determined by a function $f(l o a d)$ defining how power consumption varies with the load handled by the device. Such parameters are obtained through a one-time measurement during the initialization phase of the system, or when a new device is plugged to the PDU.

(ii) The Network Management System and the logic behind it, as discussed in the previous section, suits as a guideline through series of states that stands on the energy monitoring process. The logical assumption is to follow the impact that a device is having on the energy consumption of the whole network, since a possible anomaly during the usage phase could cause additional consumption on the other devices part of the network.

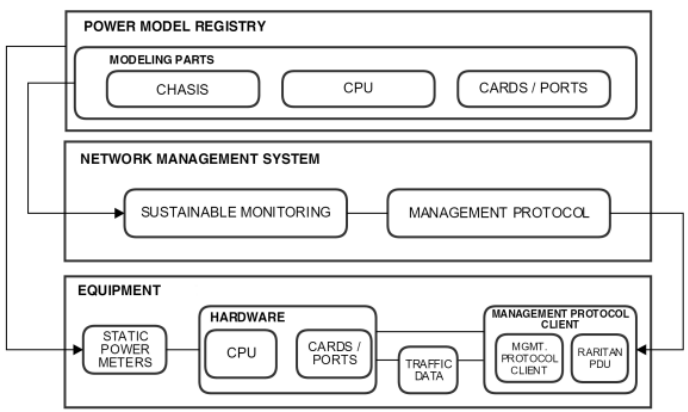

Fig.3 The architecture of the system 
(iii) $S N M P$ as an application-layer protocol that is widely accepted for managing and monitoring network elements is pinned as the first state of the execution process on Figure 2. The Raritan Power Distribution Unit used for evaluating the proposed solution comes with a bundled SNMP agent and energy MIB. Therefore, it enables easy and lightweight communication with the developed platform considering the fact that only the values for the power data from the attached equipment are fetched. Having a power distribution unit like Raritan enables following and reporting for plugged 8 to 48 devices.

\section{B. Case study}

List 1 represents the faults which are chosen as most common during a typical enterprise workday, tested on a various heterogeneous combinations of network architectures, prior building the knowledge base. To identify where the faults are applied, a classification based on the objective is depicted on the Figure 4.

List 1: faults to be detected on the network

(i) Testing the operational vs. the sleeping mode on the devices such as Cisco switches, routers, access points, Dell PCs and LCD monitors and HP laptop computers

(ii) Turning off/on a Cisco switch port

(iii) Performing link adaptation on the Cisco switch's ports

(iv) Evaluating the effect on Energy-Efficient Ethernet (EEE) and Cisco EnergyWise

(v) Testing a Spanning Tree Protocol (STP) reevaluation on the network

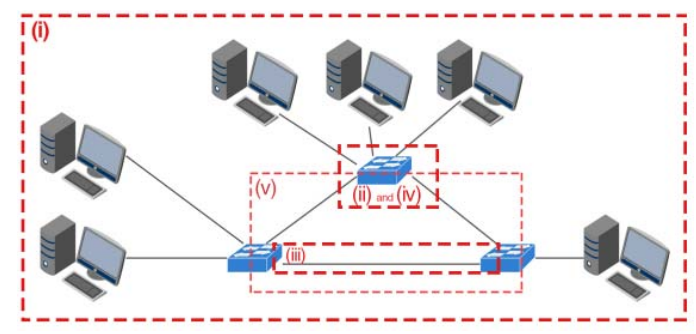

Fig.4 Classification of the objectives

One possible case study of network architecture is depicted on Figure 5, where the generated faults are represented as the ' $Y$ ' input parameter. At disposal for the experiments there were 8 Cisco switches from the series 2960 and 3560, 8 Cisco 1941 Routers, 10 Dell Precision T1700 stations, 2 HP Pavilion laptops, 6 Raritan PX2 PDUs and 2 Raritan PX3 PDUs.

(i) As suggested in [13], desktop computer are rarely turned off when they are not in use, and even though the green computing research community has focused on solving the problem of an idle PCs, the intuition is that there is still a room for improvements. For instance, the developed platform has the possibility to automatically detect the working hours of an enterprise, and by that to be able to determine if the equipment was left in the operational state. Hence, a suggestion for turning off, sleep or hibernate state is issued. Another use case is a constant level of energy consumed during non-working hours by wireless access point can indicate that the device is powered, but it does not transmit any data already for some time, meaning that it should transition into a sleep/hibernate mode.

(ii) Managing the active ports on a switch is one important and exquisite objective because it is the only way of providing network accessibility. Therefore, to have an overview on the status of the ports at one point of the time by observing the switch's values for the energy consumption is a delicate task. Mainly because of the introduction of EEE as a set of enhancements able to dynamically put a single link temporarily to sleep when not in use. One way to accomplish this is by following the consumption during the transition between shutting down and waking up a switch port, so that the platform can keep track of it and develop its own matrix of the status of the ports.

(iii) Similarly, the utilization of the Ethernet links is, on average, extremely low [16]. This suggests that there is an ample opportunity for energy savings by operating Ethernet links at a low data rate for most of the time with no perceivable performance impact to the user. Keeping track of the port's assigned speed by following the consumption of the switch is deriving a suggestion when to change the port's rate. But it is also helping the system to correlate it with the congestion, or the load on the switch, which is as well affecting the overall power consumption.

(iv) The listed energy-efficient possibilities are mostly applicable to a network switch. Cisco 2960 and 3560 switch series were available during the execution of the experiments, thus a evaluating of EEE and Cisco EnergyWise technology were at disposal for testing. Since those technologies could operate dynamically without assistance of the administrator, the developed solution has to identify and report for their changes. (v) Spanning Tree Protocol (STP) is commonly used Layer 2 protocol that runs on switches and bridges, with purpose to ensure that there are no loops created on a redundant path in the network. By default, STP dynamically manages the elimination of the loops with the process of electing a tree based on priorities with a particular switch as a root. This means that there is a possibility of a network reconfiguration without the assistance of the operator, which could lead to additional power demands if it is not well managed. To observe how STP affects the energy consumption, an STP re-evaluation has to be generated that could be performed either by physical or logical events, such as physically removing a cable from a switch or by changing the priority id on the switches.

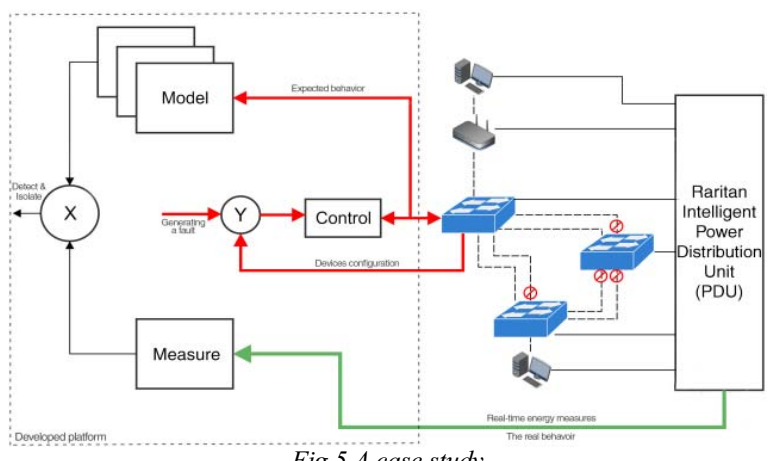

Fig.5 A case studv 


\section{Implementation}

The platform, which includes the power models and the monitoring module is implemented in Java language. The energy monitoring task is developed in a multi-threaded fashion to periodically pull the energy values from the Raritan PDU, by importing Java libraries for SNMP to access the functions for requesting the right Raritan's MiB OIDs. There is an ability to optimize the frequency of the probes send to the Raritan PDU throughout a developed GUI, thus the accuracy of the measures are practically defined by the operator. During the test phase of the platform, probes were requested every second and the responses are stored locally with the interest to enable further analysis on the data. The response from each probe is a group of values in the following format:

$$
\begin{gathered}
I_{(A)}-\text { Electric current significant with } 10^{-3} \text { amps (A) base } \\
V_{(V)} \text {-Voltage } \\
P F-\text { Power factor }
\end{gathered}
$$

Moreover, the probe response does not only contain data for the total energy consumed by the whole PDU, but also it reports the consumption of its sockets individually. This means that the platform with additional analysis can successfully build its own schemas and detect what kind of device is connected to a certain socket. Algorithm 1 describes the process flow of the platform.

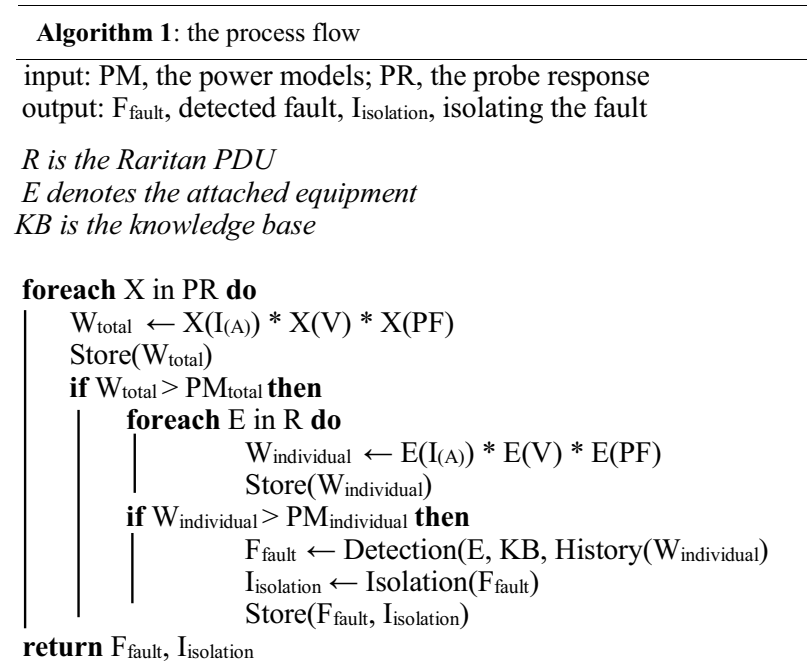

The support of power models (PM) in the platform creates the premises to set the expected threshold regarding the energy spent by each detected device. The models from [10-14] are represented with a static parameters gathered by one-time measurement at the initialization phase of the platform or when a new device is plugged to the PDU socket.

Having such real-time availability probes enables the platform to perform parallel comparison on the retrieved data for individual sockets and as a whole with the data provided by the power models. In case of aberrant values, the platform tries to locate the device with the unexpected behavior, load the latest stored power data history of the device and perform analysis for fault detection. The isolation process is based on calculations guided by the predefined fault foreknowledge achieved by the experiments. They are also integrated into the platform as a knowledge base. More about the results from this process is discussed in the next section.

\section{RESUlTS AND DisCUSSIONS}

This section refers to the List 1, presenting the obtained results during the test phase of the developed platform. Each of the experiments were tested on different network architectures, monitored for 10 minutes continuously, as well as under different amount of load generated to flow through the network.

(i) The possibility to automatically detect the working hours of an enterprise allows the platform dynamically to report on the equipment that was left in an operational state. The cost of powering the hardware components of each device dominates the overall power profile and therefore the opportunity for energy savings are the highest. The difference in the energy consumption is illustrated on the Figure 6. Typically, there is a burst of energy consumption when a device goes to operational state, either from sleep/hibernate or offline mode. Those values are also used to benchmark the current state of the device.

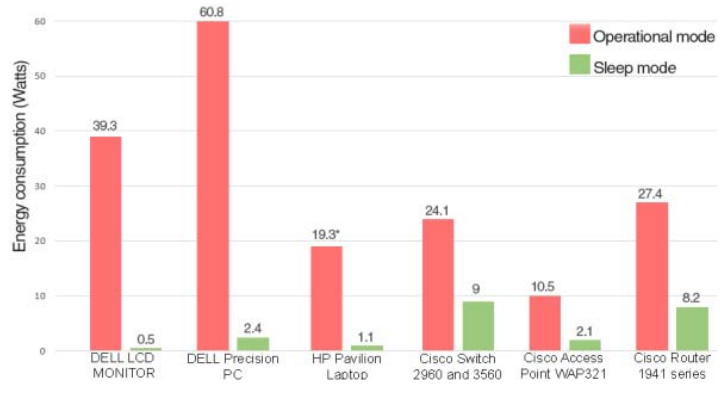

Fig.6 Obtained results from Raritan PDU - (i) experiment

(ii) The difference in the power consumption after a transition from active to inactive Cisco switch port is observed to be from 0.3 to $0.4 \mathrm{~W}$, as illustrated on the Figure 7 . This result allows the developed platform to have detailed matrix of the port statuses of each switch, which is used to report possible unnecessarily active ports, but also is part of the analysis for a possible misconfiguration of the network.

(iii) There is no notable change in the consumption when comparing $10 \mathrm{Mb} / \mathrm{s}$ to $100 \mathrm{Mb} / \mathrm{s}$ speed link, but the difference when making a transition from $1 \mathrm{~Gb} / \mathrm{s}$ to $100 \mathrm{Mb} / \mathrm{s}$ is from 0.2 to $0.4 \mathrm{~W}$ per port. Thus, the developed platform is forming a schema of each port link speed that is used as part of the suggestions for the speed reduction if a particular link is under low utilization.

(iv) Energy-Efficient Ethernet is not yet a feature supported by many network devices currently on the market, however it is important to observe its impact on the energy consumption. During an idle period of a switch with no traffic flowing through the ports, EEE will put all of the active ports in the Low Power Idle (LPI) state. Therefore, EEE is achieving the same result as the second (ii) experiment of 0.3 to $0.4 \mathrm{~W}$ savings per Cisco switch port. Hence, the developed platform has to be aware of the possibilities of EEE to dynamically, for a temporary time, put a port to idle mode.

(v) STP, enabled between the switches on the Figure 5, does not save any energy with its ability to block certain port in order to discard the loops in the network. Namely, a port in a blocking state is consuming the same amount of $0.4 \mathrm{~W}$ as the other active ports. The only noticeable difference is the spike of energy 


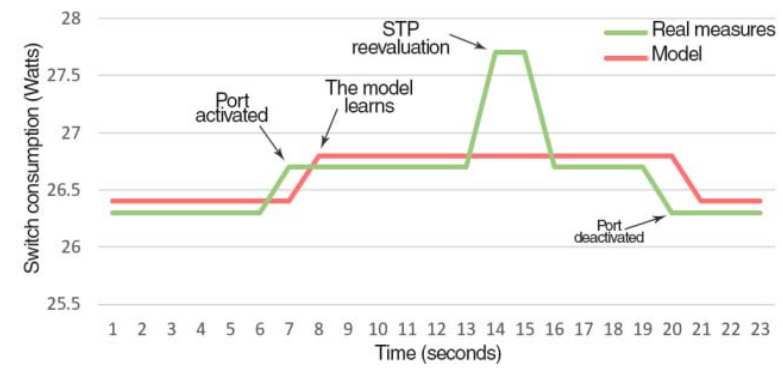

Fig.7 Obtained results from Raritan PDU - (ii) and (v) experiments

consumption when STP is trying to re-evaluate the tree. Namely, during a physical or a logical generated error, STP will elect a new root and construct the new tree. Thus, this process adds up to $1 \mathrm{~W}$ for few seconds to the overall consumption of the Cisco switch, depending on the architecture. This burst of energy, also shown on the Figure 7, is used to detect a change in the STP, with the interest of reporting it for possible misconfiguration of the network.

\section{CONCLUSION AND FUTURE WORK}

We presented results from evaluating the proposed novel form of energy-efficient NMS emulated in a heterogeneous network environment, showing that a significant power could be saved with minor impact on the network regarding the traffic. Precise figure on the energy conservations is difficult to be drawn due to the factors such as the size of the SNMP packet, number of nodes that a SNMP packet has to traverse, complexity of the network architecture and the number of MIB values required for calculation. Also, each packet as it is traversing a node invokes energy consumption for procession, memory and bandwidth usage. The size of an SNMP packet varies from 484 to 1500 bytes [21], and according to [22] $10 \mathrm{~nJ}$ is used for transferring a bit through a network. The approach of monitoring the power consumption directly from the PDU, instead of pointto-point communication with every device in conventional NMS brings own complexity as the network grows. For instance, in a set-up of multiple faults happening simultaneously, the FDI concept presented is this paper is able to cope with the occurring situation. However, this study considers an ideal scenario without testing the responsiveness of the models under noise, excluding the sensibility issues of the used models.

As a first work in the area of augmenting the extracted power data from the ICT devices, the results from this study demonstrates a promising start in the future development of energy-efficient network management systems. However, the next step would be improving the sensibility of the models, in order to be more responsive to modeling errors and noise on the network when they transpire. As future work, we envision improvements in the proposed platform by doing selfrecuperation and autonomous reconfiguration of the network, through the suggestions that are currently being produced. The anticipation is also to include other common network anomalies and to follow their impact on the energy consumption, for instance the packet loss rate and highly congested networks, which could possibly trigger a use of other techniques in order to gather all the necessary information for the analysis process, rather than just monitoring the energy values.

\section{ACKNOWLEDGMENT}

This research was supported by Erasmus+ PERCCOM (Pervasive Computing and Communications for Sustainable Development) Program.

\section{REFERENCES}

[1] Cisco, How the Next Evolution of the Internet Is Changing Everything, White Paper, April 2011

[2] Maria Kazandjieva, Brandon Heller, Omprakash Gnawali, Philip Levis, Christos Kozyraki, 2013, "Measuring and analyzing the energy use of enterprise computing systems".

[3] Vermesan, et al. Internet of things: converging technologies for smart environments and integrated ecosystems. River Publishers, 2013

[4] Koutitas, G. "Challenges for energy efficiency in local and regional data centers." (2010).

[5] Wang, Xiaofei, et al. "A survey of green mobile networks: Opportunities and challenges." Mobile Networks and Applications 17.1 (2012): 4-20.

[6] Gupta, Maruti, and Suresh Singh. "Greening of the Internet." Proceedings of the 2003 conference on Applications, technologies, architectures, and protocols for computer communications. ACM, 2003.

[7] Zhang, et al. "GreenTE: Power-aware traffic engineering." Network Protocols (ICNP), 2010 IEEE International Conference on. IEEE, 2010.

[8] Drouant, N., Rondeau, E., Georges, J.P., Lepage, F., 2014. Designing green network architectures using the ten commandments for a mature ecosystem. Comput. Commun. 42, 38-46

[9] Foll, L.S., 2008 TIC et Energetique: techniques d'estimation de consommation sur la hauteur, la structure et l'evolution de l'impact des TIC en France (Ph.D. thesis), Institut national des telecommunications.

[10] P. Reviriego, V. Sivaraman, Z. Zhao, J. A. Maestro, A. Vishwanath, A. Sánchez-Macian and C. Russell, 2012, " An Energy Consumption Model for Energy Efficient Ethernet Switches"

[11] Hossain, Md Mohaimenul, et al. "Modeling the power consumption of Ethernet switch." International SEEDS Conference 2015: Sustainable Ecological Engineering Design for Society. 2015.

[12] Ahn, Jaewon, et al. "Measurement and modeling the power consumption of router interface." Advanced Communication Technology (ICACT), 2014 16th International Conference on. IEEE, 2014.

[13] Agarwal, Yuvraj, et al. "Somniloquy: Augmenting Network Interfaces to Reduce PC Energy Usage." NSDI. Vol. 9. 2009

[14] M. Ö Demir, et al, "An energy consumption model for 802.11ac access points," Software, Telecommunications and Computer Networks (SoftCOM), 2014 International Conference on, Split, 2014, pp. 67-71.

[15] Chaudhari, Santosh K., et al. "GREEN-IT: An approach to energy savings using energy aware network management system." Communications (NCC), 2012 National Conference on. IEEE, 2012.

[16] C. Gunaratne, K. Christensen, B. Nordman and S. Suen, "Reducing the Energy Consumption of Ethernet with Adaptive Link Rate (ALR)," in IEEE Transactions on Computers, vol. 57, no. 4, pp. 448-461, April 2008

[17] Chang, Yanan, et al. "Design and implementation of NETCONF-based network management system." Future Generation Communication and Networking, 2008. FGCN'08. Second International Conference on. Vol. 1. IEEE, 2008

[18] Hedstrom, et al. Protocol Efficiencies of NETCONF versus SNMP for Configuration Management Functions. Diss. Ph. D. thesis, Masters thesis, University of Colorado, 2011.

[19] Aubrun, Christophe, et al. "Network calculus based fault diagnosis decision-making for Networked Control Systems." Emerging Technologies and Factory Automation, 2008. ETFA 2008. IEEE International Conference on. IEEE, 2008.

[20] Riekstin, et al. "Orchestration of energy efficiency capabilities in networks." Journal of Network and Computer Applications 59 (2016): 7487.

[21] Stevenson, David James, et al. "Method and apparatus for fetching sparsely indexed MIB tables in managed network systems." U.S. Patent No. 6,766,367. 20 Jul. 2004.

[22] Baliga, Jayant, et al. "Energy consumption in optical IP networks." Journal of Lightwave Technology 27.13 (2009): 2391-2403. 